\title{
Corela
}

Cognition, représentation, langage

HS-35 | 2022

Anaphore et pronoms en anglais : convergences, différences et complémentarité de quelques approches linguistiques

\section{Les pronoms de l'anglais : approche diachronique}

\section{Catherine Delesse}

\section{(2) OpenEdition}

\section{Journals}

Édition électronique

URL : https://journals.openedition.org/corela/14224

DOI : $10.4000 /$ corela. 14224

ISSN : 1638-573X

Éditeur

Cercle linguistique du Centre et de I'Ouest - CerLICO

\section{Référence électronique}

Catherine Delesse, «Les pronoms de l'anglais : approche diachronique », Corela [En ligne], HS-35 2022, mis en ligne le 03 janvier 2022, consulté le 24 janvier 2022. URL : http://

journals.openedition.org/corela/14224 ; DOI : https://doi.org/10.4000/corela.14224

Ce document a été généré automatiquement le 24 janvier 2022.

\section{(c) (1)(2)}

Corela - cognition, représentation, langage est mis à disposition selon les termes de la licence Creative Commons Attribution - Pas d'Utilisation Commerciale - Partage dans les Mêmes Conditions 4.0 International. 


\title{
Les pronoms de l'anglais : approche diachronique
}

\author{
Catherine Delesse
}

\section{Le vieil-anglais, héritage de l'Indo-Européen}

1 Commençons par cette citation de Wright (1961:238-9) sur l'origine des pronoms :

The most difficult chapter in works on comparative grammar is the one dealing with the pronouns. It is impossible to state with any degree of certainty how many pronouns the parent Indogermanic language had and what forms they had assumed at the time it became differentiated into the various branches which constitute the Indogermanic family of languages.

2 Wright poursuit en indiquant que la plupart des pronoms - particulièrement les démonstratifs et les personnels - devaient avoir une forme accentuée et une inaccentuée, et que l'une ou l'autre de ces formes « became generalized already in the prehistoric period of the individual branches of the parent languages » (id.); plus tard ont émergé de nouvelles formes accentuées et inaccentuées. Les langues germaniques bien avant les sources écrites - ont généralisé une de ces formes, et le processus s'est répété plusieurs fois. Il donne l'exemple du VA ${ }^{1} i \dot{c}$ (I en AC), qui est devenu en MA un ich accentué à côté d'un $i$ inaccentué, le premier a disparu et $I$ fut ensuite utilisé de manière accentuée et inaccentuée.

\section{Les pronoms personnels}

3 La plupart des formes personnelles du VA sont des formes inaccentuées du germanique primitif. L'exemple ci-dessous offre un bel échantillon de pronoms, incluant l'indéfini many ainsi qu'un relatif.

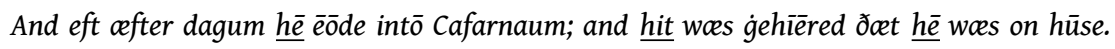

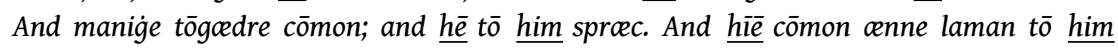

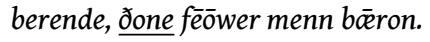


"And again he entered into Capernaum after some days; and it was noised that he was in the house. And straightaway many were gathered together, [...] and he preached the word unto them. And they come unto him, bringing one sick of the palsy, which was borne of four." (Marc II, 1-32)

\begin{tabular}{|c|c|c|c|c|c|c|c|c|}
\hline \multicolumn{9}{|c|}{ Pronoms personnels du $\mathrm{VA}^{3}$} \\
\hline & $\mathrm{Sg}$ & Dual & Plur. & cas & & \begin{tabular}{|l|} 
P3 \\
Masc.
\end{tabular} & $\begin{array}{l}\text { P3 } \\
\text { Nt. }\end{array}$ & $\begin{array}{l}\text { P3 } \\
\text { Fém. }\end{array}$ \\
\hline \multirow[t]{4}{*}{ P1 } & $\mathrm{ic}$ & wit & $w \overline{\mathrm{e}}$ & Nom. & \multirow[t]{4}{*}{$\mathrm{Sg}$} & $\mathrm{h} \overline{\mathrm{e}}$ & hit & hēo \\
\hline & $\mathrm{me}$ & unc & $\overline{\mathrm{u}} \mathrm{s}$ & Acc. & & hine & hit & hie \\
\hline & $\operatorname{mīn}$ & uncer & ūre & Dat. & & \multicolumn{2}{|c|}{ his } & $\begin{array}{l}\text { hiere, } \\
\text { hire }\end{array}$ \\
\hline & $\mathrm{me}$ & unc & $\overline{\mathrm{u} s}$ & Gén. & & & $\mathrm{m}$ & \\
\hline \multirow[t]{4}{*}{ P2 } & ðū & git & $\dot{\mathrm{g}} \overline{\mathrm{e}}$ & Nom. & \multirow[t]{4}{*}{ Plur. } & \multirow{2}{*}{\multicolumn{3}{|c|}{ hìe }} \\
\hline & 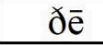 & inc & ēow & Acc. & & & & \\
\hline & Ø̄in & incer & ēower & Dat. & & \multicolumn{3}{|c|}{ hiera } \\
\hline & 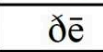 & inc & ēow & Gén. & & \multicolumn{3}{|c|}{ him } \\
\hline
\end{tabular}

Tableau 1 : Pronoms personnels du vieil-anglais

4 Les pronoms personnels du VA ont évidemment beaucoup évolué :

5 - le duel n'existe plus en $\mathrm{AC}: \mathrm{le}<\mathrm{t}>\mathrm{de}$ wit/git est d'origine obscure mais pourrait être relié au numéral deux (m. twēgen; f. twa) ;

$6-\mathrm{unc} / \mathrm{pec} / \mathrm{mec}$ le $<\mathrm{c}>$ dans ces accusatifs remonte à une particule germanique primitive et emphatique *ke (Indo-européen *ge). On ne trouve ces formes que dans les écrits les plus anciens et en poésie. Ic est l'ancienne forme inaccentuée (la forme accentuée étant préservée par le vieil islandais ek, voir aussi le latinego). Unc/inc sont d'anciens accusatifs utilisés au datif. Campbell (1991 : 288) nous indique par ailleurs que ces formes en $-c$ sont fréquentes en Northumbrien, dialecte du Nord ;

7 - hit, le pronom neutre, a donné it.

8 Outre des évolutions graphiques dues en partie aux scribes anglo-normands, nous assistons à la période moyen-anglaise à une simplification des finales, des déclinaisons, à des emprunts et à des phénomènes de grammaticalisation (par exemple les pronoms interrogatifs qui développent un emploi relatif).

9 Mossé (1959: 84) indique que «la flexion des pronoms qui, en vieil-anglais, était relativement riche, a subi une simplification assez grande en moyen-anglais, mais elle ne s'est réalisée que petit à petit. Le trait le plus net est l'élimination complète de l'accusatif au profit du datif qui, désormais, sert de cas régime en face du nominatif », ceci ayant déjà commencé en VA. Selon Lass (2006 : 72), «In early ME the masculine accusative was still retained in the west, but not in the (usually more advanced) east: the Peterborough Chronicle in the twelfth century has already merged the two under the old dative ". Il indique également la perte, en MA, du duel qui a cependant perduré jusqu'au XII ${ }^{\mathrm{e}}$ XIII ${ }^{\mathrm{e}}$ siècles. "En outre, dans le pronom personnel, l'ancien génitif disparaît de bonne heure en moyen-anglais, en tant que tel, et prend la fonction de pronom possessif. » (Mossé id.) 


\begin{tabular}{|c|c|c|c|}
\hline & & Singulier & Pluriel \\
\hline \multirow[t]{4}{*}{$\mathrm{P} 1$} & Sujet & $\begin{array}{l}\text { ich (forme accentuée } \\
\text { Sud/Midlands) }\end{array}$ & $w \bar{e}$ \\
\hline & & $i c, i k$ (forme accentuée Nord) & \\
\hline & & $\begin{array}{l}\text { I, y (forme inaccentué Nord }> \\
\text { emploi général langue commune } \\
\text { du XIVe) }\end{array}$ & \\
\hline & Régime & $m \bar{e}$ & us, ous \\
\hline \multirow[t]{3}{*}{$\mathrm{P} 2$} & Sujet & pu, pou & $3 \bar{e}, y \bar{e}$ \\
\hline & & $\begin{array}{l}\text { tu, tow/tou, te (forme enclitique } \\
\text { souvent accolée au verbe : } \\
\text { woltou }^{4} \text { ) }\end{array}$ & \\
\hline & Régime & $p \bar{e}$, thee, te & $\begin{array}{l}\text { eu, ou (formes anciennes) } \\
\text { zow, zou, you (forme courante < } \\
\text { XIIIe. Soit par déplacement } \\
\text { d'accent, soit par contamination } \\
\text { entre sujet ze et ou) }\end{array}$ \\
\hline
\end{tabular}

Tableau 2 : Pronoms de 1 ère et 2ème personnes en moyen-anglais

I $k$ : la consonne finale commence à tomber devant une consonne au XII ${ }^{e}$ siècle. Vers 1370 l'usage s'étend à la région de Londres, et ensuite apparaît une nouvelle forme forte avec allongement $\mathrm{du}<\mathrm{i}>$ pris dans la $\mathrm{GMV}^{3}$. La forme du sud ich / $\mathrm{t} \mathrm{t} /$ / est à l'origine du ch dialectal utilisé par T. Hardy "'ch woll not » (= I will not) (Elliott 1984 : 38-39) ${ }^{4}$.

11 La graphie $b$ de P2 cède rapidement la place à <th>, qui fournit des formes au statut marqué par rapport à «you/ye». Les formes en ge étaient uniquement plurielles en VA, le tutoiement étant utilisé pour s'adresser à une seule personne. L'apparition du vouvoiement au MA (selon l'OED, le premier exemple de ye au nominatif date de 1297) gagne progressivement du terrain : avec l'apparition d'un pluriel de politesse au XIII ${ }^{\mathrm{e}}$ siècle, les formes du singulier s'utilisent entre familiers ou pour les enfants. Selon Colette Stévanovitch (2003), ces formes indiquent une intention particulière de l'énonciateur, respect envers un supérieur ou un adulte ou mépris selon les cas. ${ }^{5}$ Thou dans la Bible de 1611 est employé pour s'adresser à un être unique, Dieu. Entre égaux on se vouvoie dans la bonne société et on se tutoie dans le peuple.

\begin{tabular}{|l|l|}
\hline $\begin{array}{l}\text { Chaucer, The Canterbury Tales, The } \\
\text { Pardoner's Tale. Fin XIV }\end{array}$ & $\begin{array}{l}\text { Trad. André Crépin (Le Vendeur } \\
\text { d'indulgences), 2000, Folio. }\end{array}$ \\
\hline 'Sire, ' quod this boy, 'it nedeth never-a-deel ; & $\begin{array}{l}\text { Ce n'est pas la peine, Monsieur, répondit-il } \\
\text { [le jeune valet]. } \\
\text { Deux bonnes heures avant que vous n'arriviez } \\
\text { On me l'a dit : c'est l'un de votre groupe } \\
\text { (p. 395) }\end{array}$ \\
$\begin{array}{l}\text { It was me toold er ye cam heer two houres. pardee, an old felawe of youres; } \\
(670-672)\end{array}$ & $\begin{array}{l}\text { Approche, Hôtelier ; mets d'abord ton } \\
\text { offrande } \\
\text { Et tu embrasseras toutes les reliques, } \\
\text { Tout ça pour un liard. Vite, dégrafe ta bourse. } \\
\text { (p. 403) }\end{array}$ \\
$\begin{array}{l}\text { And thou shalt kisse my relikes everychon grote! Unbokele anon thy purs.' } \\
(943-945)\end{array}$
\end{tabular}

Pour la Renaissance, Stévanovitch (2003) propose cette distinction: à un premier niveau de signification, le vouvoiement est une forme non-marquée utilisée d'inférieur à supérieur, ou autres égaux de rang élevé; le tutoiement une forme non marquée entre personnes de rang inférieur ou de supérieur à inférieur. Les notions de respect (ye/you) ou de familiarité (thou) sont d'ailleurs très nettes chez les auteurs élisabéthains. Stévanovitch (2003: 430) propose le tableau suivant : 


\begin{tabular}{|l|l|l|l|}
\hline & $\begin{array}{l}\text { Interlocuteur } \\
\text { supérieur }\end{array}$ & Interlocuteur égal & Interlocuteur inférieur \\
\hline $\begin{array}{l}\text { Locuteur de rang } \\
\text { élevé }\end{array}$ & you & you & thou \\
\hline $\begin{array}{l}\text { Locuteur de basse } \\
\text { condition }\end{array}$ & you & thou & thou \\
\hline
\end{tabular}

Tableau 3 : Tutoiement et vouvoiement à la Renaissance

Quand le discours s'adresse à un objet, une abstraction ou une divinité, une personne absente ou morte, voire au locuteur lui-même dans un monologue, on utilise le tutoiement. ${ }^{6}$ Mais il peut y avoir une coloration émotionnelle: le tutoiement émotionnel est un choix individuel qui va à l'encontre des règles sociales. Vouvoyer quelqu'un qu'on devrait tutoyer indique une distance respectueuse ou glaciale.

14 Le PP sujet est généralement exprimé en MA; on peut avoir un sujet d'anticipation quand le sujet est rejeté loin derrière le verbe. Le sujet est parfois inexprimé dans un dialogue rapide et familier, ou en poésie; dans les propositions coordonnées ou juxtaposées à sujet identique et dans les tournures impersonnelles, très nombreuses en MA (remarquer la tournure utilisée dans l'exemple ci-dessus - souligné - équivalent de me was toold).

5 En ce qui concerne you/ye, il y avait une distinction au départ entre nominatif et accusatif, mais comme ces formes étaient généralement non accentuées, elles étaient prononcées /jə/ et au XIV siècle on a commencé à employer you au nominatif. Selon Denison (1993 : 22), vers la fin du XVI ${ }^{e}$, il y avait confusion entre le - historiquement subjectif ye et l'objectif you: il note une tendance dans la langue à un glissement du subjectif vers l'objectif comme en témoigne l'emploi contemporain de it's me / taller than me au lieu de It's I / taller than I.

Nevalainen (2009: 562-563) indique pour sa part que ce remplacement de ye par you fut assez rapide, comme le montre la figure ci-dessous.

Throughout the Early Modern period you vastly outnumbers thou in personal letters, which reflects everyday language use. The contexts where thou typically occurs in seventeenth-century correspondence include a mother writing to her child, or spouses expressing their mutual affection. Even these writers alternate between the two pronouns within one and the same letter. (Nevalainen $2006: 79$ ).

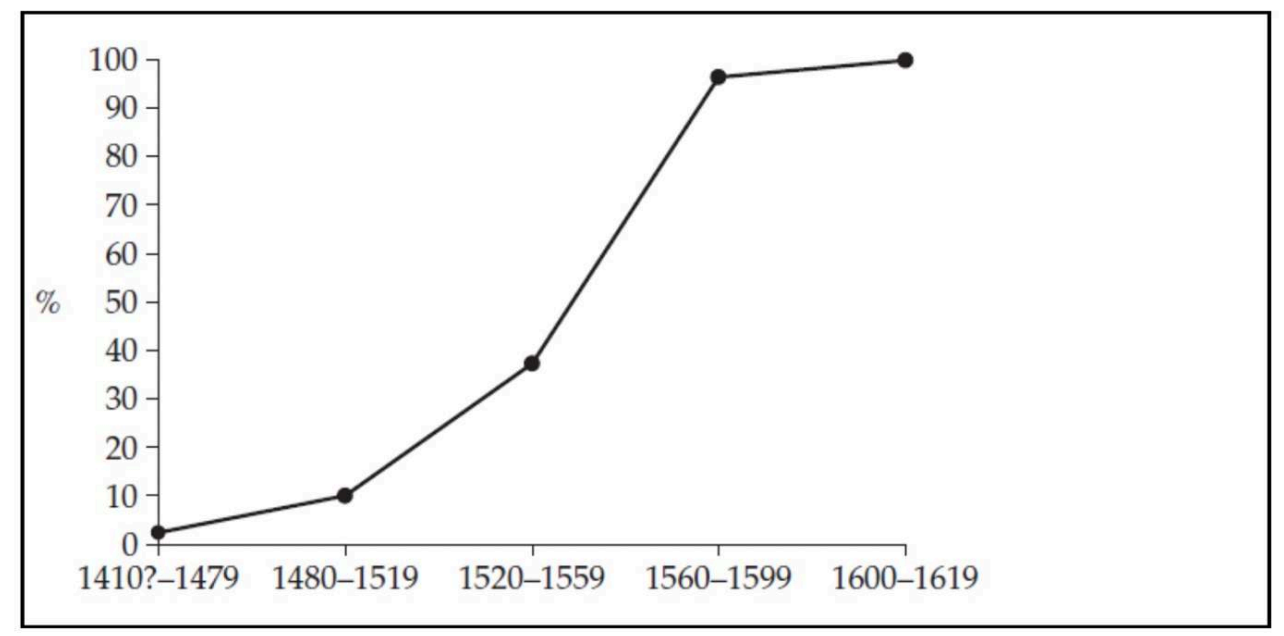

Figure 1 : Remplacement de ye par you en position de sujet : pourcentages de you (Nevalainen 2009 : 563) 
Cependant Wright (1961) note que cette distinction ancienne persiste dans certains dialectes au XIX $19^{\text {ème }}$ siècle, ce qu'indique également Chevillet (1991: 83) : thou et thee se maintiennent dans le sud-ouest pour le tutoiement, thou en position sujet et thee en position objet et même sujet comme en témoignent ces exemples tirés de Thomas Hardy $^{7}$ :

'Well, travellers,' he said, 'did I hear ye speak to me?'

'You did: you've got to come and be our prisoner at once!' said the constable. 'We arrest 'ee on the charge of not biding in Casterbridge jail in a decent proper manner to be hung to-morrow morning. Neighbours, do your duty, and seize the culpet!'

(“The Three Strangers", Wessex Tales (1965)[1888], London: Macmillan, p.26)

C'est la troisième personne qui voit le plus de changements, notamment pour le pluriel et la $3^{\text {ème }}$ personne au féminin singulier. Pour le pluriel, il s'agit d'un emprunt des dialectes du Nord et de l'écossais au VN. L'Ormulum (environ 1200) ne connaît que ces formes en $b$; le sous-système a été entièrement emprunté. Lass (2006) indique qu'il a fallu 400 ans pour que le nouveau paradigme s'installe dans la variété dialectale et devienne la norme en anglais standard. Le Nord a progressivement contaminé les Midlands et Londres. Selon Mossé - à qui j'emprunte les tableaux ci-dessous (1959 : 85) - them est inconnu à Londres, où l'on employait hem ${ }^{8}$, avant le $\mathrm{XV}^{\mathrm{e}}$ siècle.

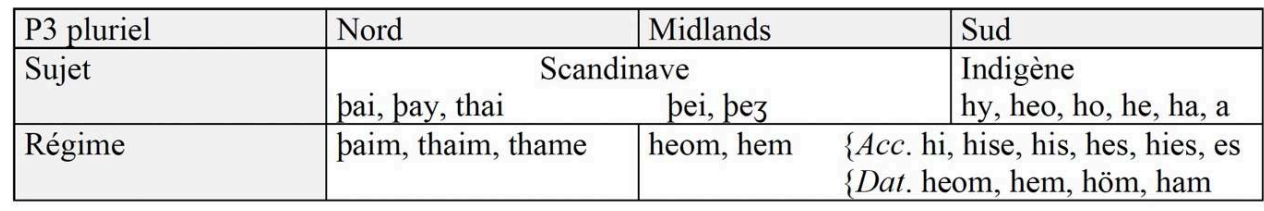

\begin{tabular}{|c|c|c|c|c|}
\hline \multicolumn{2}{|c|}{ P3 singulier } & $\mathrm{M}$ & $\mathrm{Nt}$ & $\mathrm{F}$ \\
\hline \multicolumn{2}{|c|}{ Sujet } & hē, hee, ha, a & hit, it, a & $\begin{array}{l}\text { 1. heo (Ouest), hue, } \\
\text { ho, he, ha, hi } \\
\text { 2. zho (NO } \\
\text { Midlands), cho, scho } \\
\text { 3. sche, she }\end{array}$ \\
\hline \multirow[t]{2}{*}{ Régime } & Acc. & hine, hin & hit, it & hire, hir, hure \\
\hline & Dat. & him & him & \\
\hline
\end{tabular}

Tableaux 4 et 5 : Pronoms personnels de 3ème personne en moyen-anglais

Le cas le plus compliqué est l'origine du pronom she pour lequel plusieurs hypothèses sont avancées. Le premier exemple attesté est scce en1140 dans la Peterborough Chronicle.

[...] she was invented, and although we are not sure why (if that is even an askable question), we can ask how. That puzzle has not yet been solved to everybody's satisfaction. The sources invoked are normally either the feminine determiner sēo or the nominative singular pronoun hēo. Both are difficult but not impossible. (Lass $2006: 74)$

Première hypothèse : sēo : transfert d'accentuation [séo] > [seó], puis réduction de [e] à [j] et allongement du [o] [seó] > [sjo:] et palatalisation de [sj] donnant [Jo:] ; selon Lass, c'est cohérent au niveau phonétique bien que ce ne soit pas la bonne voyelle pour le sud où il faudrait que [e:] soit un transfert analogique depuis hēe ce qui est d'après lui plausible puisque cela forme une paire qui rime.

Deuxième hypothèse pour hēo: la "Shetland theory" ainsi nommée en raison du parallélisme entre Shetland et le veiux scandinave Hjatland. [héo] > [heó] > [hjo:], puis [hj] > [ç] qui devient [S]. Il faut le même transfert analogique pour obtenir la bonne 
voyelle, mais selon Lass le fait que la source soit un pronom personnel plutôt qu'un déterminant, ainsi que les exemples lexicaux et/ou de noms de lieux comme Shetland en font un bon candidat. Il reste tout de même un problème pour Lass : she apparaît d'abord de façon sporadique au milieu du XII ${ }^{\mathrm{e}}$, or les diphtongues originales du VA se sont monophtonguées au XI'. Lass conclut de façon un peu lapidaire, "Whatever the facts of the matter, it seems likely that hēo or sēo or both are somehow involved. » (Lass $2006: 74$

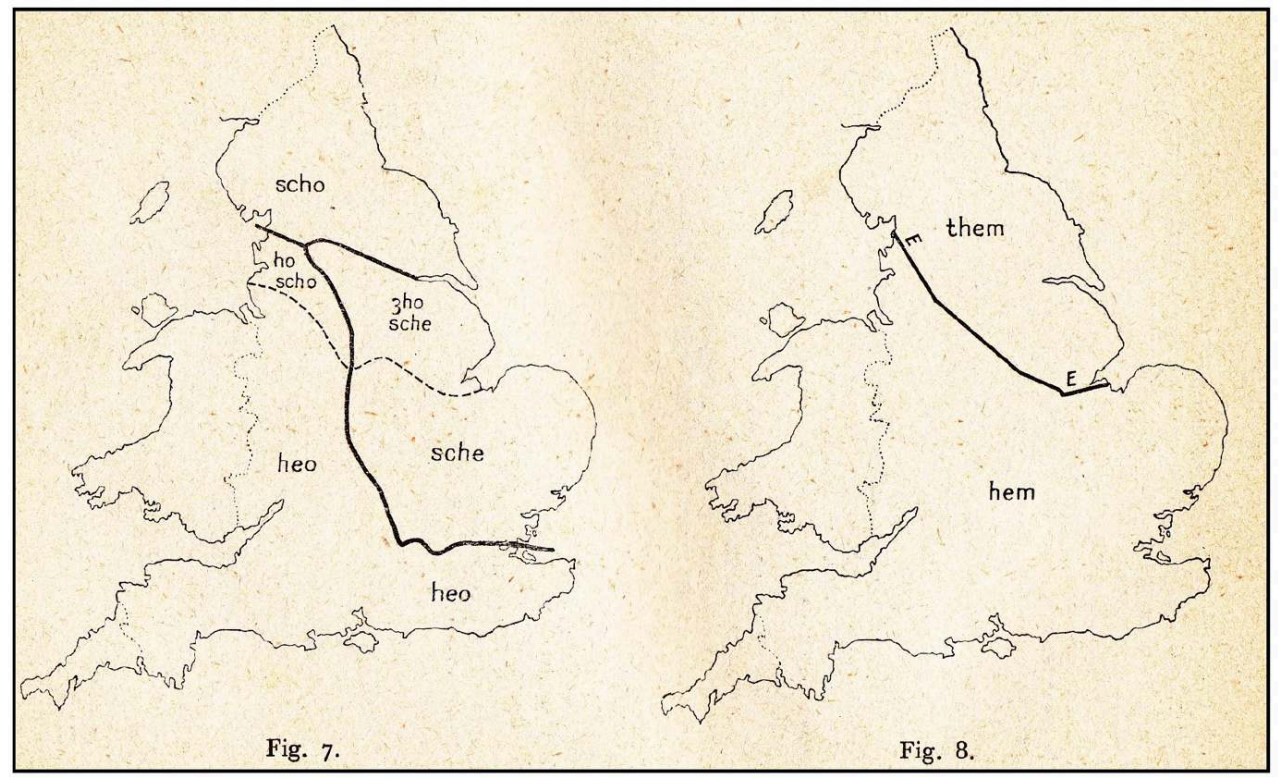

Figure 2 : Répartition dialectale des formes des pronoms personnels de $3 e \mathrm{p}$. en moyen-anglais (Mossé 1959: 87)

\section{Les pronoms possessifs}

Les formes de génitifs des pronoms personnels ont donné le pronom possessif de l'anglais : $\min >$ mine, üre > our, his > his. En VA on utilisait le cas génitif pour indiquer la possession, on n'avait donc pas de possessifs au sens où on l'entend en $\mathrm{AC}$ : on disait min sune = littéralement «de moi le fils» pour «mon fils». La distinction my/mine, amorcée dès le XIV ${ }^{e}$, remonte à la période élisabéthaine.

De façon générale, on sait que le marqueur casuel -es fut rapidement étendu à tous les noms et fut plus tard ré-analysé en marqueur de cas clitique : 's. Le génitif perd du terrain au profit des structures en of ( $80 \%$ des constructions du génitif à la fin du MA tardif d'après Crystal) ce qui va de pair avec la perte générale des cas au cours de la période MA.

C'est au cours de la période MA que s'établit la distinction adjectifs (déterminants) et pronoms possessifs: en principe, nous dit Mossé, c'est l'accent qui permet de les distinguer. Le pronom est toujours accentué. L'adjectif peut être antéposé ou postposé, ce dernier peut être renforcé par owen: He hadde no house of His owene (...). (Wyclif, Ms 296 de Corpus Christi College, Cambridge, XIV) (he had no house of his own).

Les formes nouvelles hers, ours, youres, thaires sont toujours postposées. Ces formes en re apparaissent d'abord dans le Nord au XIII ${ }^{\mathrm{e}}$ siècle et gagnent les Midlands dans la seconde moitié du XIV ${ }^{e}$ siècle et le sud à la fin du XIV ${ }^{e}$ siècle. On commence à utiliser 
des formes comme "an old felawe of yours" (an old fellow of yours) (Chaucer, The Pardoner's Tale, cité p.4).

\begin{tabular}{|c|c|c|c|c|}
\hline & P1 & P2 & \multicolumn{2}{|c|}{ P3 } \\
\hline & & & Masc-Nt & Fém \\
\hline Singulier & $\min , \operatorname{mi}$ & pīn, pī, thý & $\begin{array}{l}\text { his, hise, } \\
\text { hies, hys, } \\
\text { hus }\end{array}$ & $\begin{array}{l}\text { hire, here, } \\
\text { hir, her }\end{array}$ \\
\hline Pluriel & ūr(e), ōūr(e) & zūr(e), yōūr(e), ōūre & $\begin{array}{l}\text { here, her, } \\
\text { hare, hire, } \\
\text { bezre, pay }\end{array}$ & $\begin{array}{l}\text { or, heore, } \\
\text { ure; } \\
\text { their, thar }\end{array}$ \\
\hline
\end{tabular}

Tableau 6 : Les possessifs en moyen-anglais (Mossé 1959 : 89)

His est la forme commune au M et Nt, au F hir est la plus employée jusqu'en 1400, her se répand lentement dans la langue littéraire à partir du XV $\mathrm{XV}^{\mathrm{e}}$ siècle. Its apparaît à la fin du $\mathrm{XVI}^{\mathrm{e}}$ siècle, et Roger Lass $(2006: 96)$ indique que la $1^{\text {ère }}$ occurrence écrite est répertoriée dans John Florio "for its owne sake» en 1598, la généralisation du pronom its n'intervenant qu'un siècle plus tard.

Denison (2009) rappelle que l'alternance $\mathrm{min} / \mathrm{mi}$, pin/pi dépendait de la première lettre du mot suivant (voyelle ou consonne) : extrait de Malory thyne oure (your hour/time) vs. thy grete batayle (your great battle). Cette alternance au cours de la période $\mathrm{AE}$ a amené un traitement grammatical des paires my/mine, thy/thine (et no/none) ; en même temps d'autres possessifs comme our, your, her, their avaient développé des formes en $-s$ :

These are historically double possessives, used exclusively as independent (= disjunctive) pronouns apart from brief, occasional interludes as possessive determiners in ME (hers), EModE (ours, yours) or both periods (theirs). The forms without $-s$ has wholly lost pronominal function before the end of the EmodE period, thus bringing about the same grammatical alternation, D Prn, for your yours as for my mine. Only the possessives whose determiner form ends in $-s$ already (his, its, one's, whose) have failed to develop this grammatical alternation. (Denison 2006 : 292)

\section{Les réfléchis}

Le pronom personnel à l'accusatif était utilisé pour le réfléchi en VA qui avait, selon Mossé (1950 : 79), " perdu tout pronom réfléchi ». Self, seolf, sylf, silf > self était en fait utilisé pour renforcer un substantif ou un pronom. Ce n'était pas un emploi obligatoire. Ilca (<is-lic) était utilisé avec l'article défini (the same) et survit dans le syntagme of that/ his/their ilk (= kind/type).

$\mathrm{Au}$ MA, c'est toujours le pronom personnel qui est utilisé, le MA n'a pas encore constitué de réfléchi. Le mot self (et ses variantes seolf, solf, sulf, sylf) est employé comme adjectif ou substantif. Dans le premier cas, on l'ajoute au pronom personnel ou au substantif pour le renforcer : il s'agit de formes intensives. Le réfléchi actuel est un mélange des deux formes. Le pluriel -selves date du XV $\mathrm{X}^{\mathrm{e}}$ siècle et le neutre itself date du $\mathrm{XVI}^{\mathrm{e}}$ siècle.

Par exemple dans Jacob's well (MS Salisbury 103, copié vers 1440-50, East Anglia), on trouve ces deux formes :

Placidus [...] fell doun his hors to the erthe and lay an houre out of hym self

he hastyd hym in the watyr to a fecchyd the oper chyld... 
Placidus (...) tomba de son cheval à terre et resta une heure évanoui [« hors de luimême »]

il se dépêcha d'aller dans l'eau pour chercher l'autre enfant...

31 Vers 1500 les réfléchis passeront du statut de simples pronoms à des composés en -self. L'usage du pronom personnel continue jusqu'au XVII ${ }^{\mathrm{e}}$ siècle, comme le montre cet exemple :

I am myself indifferent honest; but yet I could accuse me of such things that it were better my mother had not borne me. (Hamlet, Act III, Scene I)

\section{Les pronoms démonstratifs}

Mossé (1950) fait une distinction entre article et démonstratif, alors que Wright (1961), pour sa part, range l'article dans la catégorie «pronom démonstratif ». Florence Bourgne, qui a adapté le tableau de Mossé, a également mis cette colonne sous l'étiquette "demonstrative pronouns ». La colonne "article» du tableau de Mossé a donné the, that/those en $\mathrm{AC}$ et la colonne "démonstratif », this/these. Ils ont tous une origine démonstrative en IE : *to- pronom démonstratif; de cette racine sont issus the (Germanique *thē), that (Germ. *that), this (Germ. *thasi), there (Germ. *thēr). Fischer \& Wurff (2006) notent d'ailleurs que les démonstratifs donnent fréquemment naissance à des articles définis.

\begin{tabular}{|c|c|c|c|c|c|c|}
\hline & \multicolumn{3}{|c|}{ Article (the, that, those) } & \multicolumn{3}{|c|}{ Démonstratif (this, these) } \\
\hline & Masc. & Neutre & Fém. & Masc. & Neutre & Fém. \\
\hline Nom.sg & $\mathrm{se \overline { \textrm {e } }}$ & \multirow[t]{2}{*}{ pæt } & sēo & bes & \multirow[t]{2}{*}{ pis } & bēos \\
\hline Acc. sg & pone & & $\mathrm{p} \overline{\mathrm{a}}$ & pisne & & bās \\
\hline Gén. sg & \multicolumn{2}{|c|}{ bæs } & \multirow[t]{2}{*}{ pǣre } & pisses & & \multirow[t]{2}{*}{ pisse, pisre } \\
\hline Dat. sg & \multicolumn{2}{|c|}{ bām, pǣm } & & pissun & & \\
\hline Instr. Sg & \multicolumn{2}{|c|}{ bý, bon } & & býs & & \\
\hline Pluriel & & & & \\
\hline Nom/acc. & \multicolumn{3}{|c|}{ bā } & \multicolumn{3}{|c|}{ bās } \\
\hline Gén. & \multicolumn{3}{|c|}{ pāra, pǣra } & \multicolumn{3}{|c|}{ pissa, pisra } \\
\hline Dat. & \multicolumn{3}{|c|}{ pām, pǣm } & \multicolumn{3}{|c|}{ bissum } \\
\hline
\end{tabular}

Tableau 7 : Les démonstratifs [adaptation des tableaux de Mossé et de Bourgne]

pes, pis, pēos $(\text { pios })^{9}$ : d'après Wright (id.) ces pronoms sont en fait des composés, le démonstratif simple + une particule déictique -se/-si, dont l'usage était probablement à l'origine emphatique (voir this here en $\mathrm{AC}$ ), et qui est devenu ensuite un simple démonstratif. Ce composé existe dans toutes les langues germaniques à l'exception du gotique. Bourquin (2011: 12) pense que le -s dans cette forme indique une surdéfinition : « on est allé un nombre indéfini de fois sans rien trouver d'identique (sinon de mieux) et on va faire régulièrement retour sur le point de départ [...]».

Denison indique que l'on passe d'un système déictique à deux termes en VA se/pes à un système à trois termes en MA réorganisé autour de the - that - this.

pat en MA cesse d'être employé comme neutre de l'article pour devenir après le XIIe siècle un démonstratif singulier pour tous les genres ; pour le pluriel, on utilise d'abord celui de l'article $p a / p o$ puis peu à peu $p \bar{a} s / p o \bar{s}$ issus du VA $p \bar{a} s$. Le démonstratif du VA se maintient avec ses formes jusqu'à la fin du XII ${ }^{\mathrm{e}}$ siècle, après quoi pis dans L'EastMidlands et le Nord, pes dans le sud, vont se généraliser sans distinction de genre ou de flexion. On formera le nouveau pluriel sur ce singulier : pìse, pēse. (Mossé 1959: 92). De 
cette façon on peut observer que le paradigme casuel des démonstratifs disparaît assez tôt en MA. ${ }^{10}$

\section{Les pronoms interrogatifs}

Les pronoms interrogatifs viennent d'une racine IE ${ }^{*} k^{\mathrm{w}} \mathrm{o}$ - qui a donné des pronoms interrogatifs et relatifs. Cependant en germanique, comme nous l'indique Pons (1956: 292) le relatif se sépare de l'interrogatif, alors qu'en latin on a des formes proches, quis, quae, quid interrogatifs et quī, quae, quod relatifs.

\begin{tabular}{|c|c|c|c|c|c|}
\hline & Nom. & Acc. & Gén. & Dat. & Inst. \\
\hline Masc-Fém & hwā & $\begin{array}{l}\text { hwone, } \\
\text { hwæne }\end{array}$ & \multirow[t]{2}{*}{ hwæs } & \multirow[t]{2}{*}{ hwām, hwæm } & \multirow[t]{2}{*}{ hwý, hwon } \\
\hline Neutre & hwæt & & & & \\
\hline
\end{tabular}

Tableau 8 : les pronoms interrogatifs

On remarque que les interrogatifs en VA ont une forme unique pour le masculin et le féminin. On peut ajouter à cette liste hwilc « lequel » (formé sur le composé hwā-lï̀, ce dernier mot = «forme, corps » ayant donné like, le suffixe adverbial -ly et le lexème lichgate/lych-gate ${ }^{11}$ ) qui a donné which, et hwceðer « lequel des deux » qui a donné whether. Le VA $h w$ - deviendra wh- en MA.

Voici quelques exemples de questions en VA :

Dū, cnapa, hwoet dydest tōdøeg? « Toi, garçon, qu'as-tu fait aujourd'hui ?»

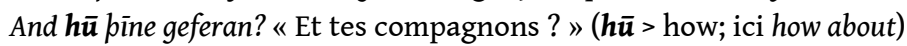

Hwōer slēpst pū? «Où dors-tu?»

Hwā āwecp pē tō ūhtsancge? "Qui te réveille pour les matines?»

(Ælfric’s Colloquy, "In the schoolroom”. Marsden 2004: 8-10)

\section{Les pronoms relatifs}

Les formes de pronoms relatifs que nous connaissons n'existaient pas en VA. « Le vieilanglais est encore dépourvu de pronoms relatifs; pour l'exprimer il a recours à divers procédés syntaxiques» (Mossé 1945: 79). Selon Wright (1961: 247), «A relative pronoun proper did not exist in primitive Germanic. The separate Germanic languages expressed it in various ways. ».

En VA, il y avait trois manières d'utiliser le relatif :

42

- le démonstratif article déclinable se, seo, poet (= la forme que retiendra le MA sous la forme du neutre that). Bourcier indique qu'il engendre des effets dissociatifs (grosso modo la relative appositive). Comme l'a montré Annie Lancri (1999: 26), il n'est pas toujours facile de trancher entre le relatif who ou bien le déictique this one:

ic habbe cnapan se sceal pa oxan mid gade pywan

J'ai un garçon de ferme qui (celui-ci ?) doit faire avancer les bœufs à la baguette

(Ælfric’s Colloquy « Dialogue des métiers »)

And hīe cōmon cenne laman tō him berende, Jone fēōwer menn bēeron

Et ils vinrent un paralytique à lui (Christ) amenant que quatre hommes portaient

(Marc 2.3) 
43 Particule indéclinable be - Pons (1956) signale qu'il s'agit probablement d'une forme affaiblie $^{12} \mathrm{du}$ démonstratif neutre poet - a des effets associatifs selon Bourcier : relation serrée (déterminative).

Hie comon ða to ðcere stowe be him gesweotolode god

Ils arrivèrent à ce lieu que leur avait indiqué Dieu (Sacrifice d'Abraham)

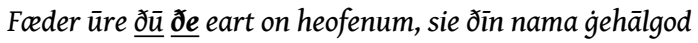

Père de nous, toi qui est dans les cieux (que) soit de toi le nom sanctifié

(Mathieu VI, 9: Our father which art in heaven. Hallowed be thy name - Bible de 1611)

Selon Denison (1993), la particule pe étant indéclinable, sans genre ni nombre, et apparaissant comme constituant de nombreuses conjonctions de subordination, on peut la considérer comme un équivalent du that moderne. Il remarque également que l'orphelinage de la préposition existait déjà en VA et donne entre autres exemples, celui-ci (id., p.130-131) :

byslic wees seo syn be se cyning fore ofslegen woes

such was the sin that the king for slain was (for which the king was slain) (Bede 3.

16. 228.5)

- la combinaison des deux : art-dém. + particule : se pe, qui avait des effets intensifs selon Bourcier ; emphase, insistance forte selon Crépin.

Eart ðu se Beowulf se be wið Brecan wunne

Are you that Beowulf who contended with Breca

(Trad. de Donaldson, Beowulf, éd. Joseph Tuso, Norton Critical Edition, 1975)

Certains voient un relatif zéro qui s'utilise en position sujet et objet jusqu'en anglais moderne, bien qu'il soit difficile de trancher en l'absence de ponctuation: est-ce le relatif qui manque ou un pronom personnel en position sujet? Pons penche pour une ellipse du relatif dans cet exemple et Stévanovitch (2008: 76) penche également pour un relatif $\varnothing$ :

Him $p \bar{a}$ andswarode his ealdorbiscop Ø Cefi wces häten

À lui alors répondit son archevêque qui (il ?) Cefi se nommait (Bede $9^{\text {ème }}$ siècle)

André Crépin signale à cet égard que le relatif $\varnothing$ s'emploie en VA et en MA surtout avec le verbe hātan « (s')appeler », ce qui est le cas dans l'exemple ci-dessus.

Selon Mossé, le pronom relatif du MA est sans conteste bat (that, thet), d'abord combiné avec se; la particule pe s'utilise jusque vers 1250 bien qu'il puisse s'agir d'un emploi particulier du nouvel article défini.

Acc bu shallt findenn batt $^{13}$ min word But you shall find that my word

E33whor poer itt iss ekedd, Anywhere where it is developed

Ma33 helpenn ba batt reden itt May help those who read it

(Ormulum, rédigé vers 1200, dédicace de l'auteur)

Le grand changement du MA est l'utilisation des interrogatifs en WH- comme pronoms relatifs, et cela dès le XII ${ }^{e}$ siècle en concurrence avec that. Selon Fischer \& Wurff (2006 : 127), il s'agit à la fois d'un développement naturel mais aussi de l'effondrement du système des relatifs du VA. Au XIV e siècle, on commence à utiliser comme relatifs des formes de pronoms interrogatifs comme which (plur. whiche) « qui, que» ou adj. « quel, lequel »; le génitif whōs et le régime whōm. Who, en revanche, reste uniquement interrogatif. Dans l'exemple ci-dessous, on note ainsi l'emploi de which, et non de who, qui serait agrammatical :

Criseyde, which that felte... (Chaucer, Troilus and Cryseide (v. 1385), III.1198)

Criseyde, qui sentit ... 
l'exemple suivant on notera que le relatif which est suivi d'un that, qui semble indiquer ici que le mot qui le précède n'est pas un interrogatif, mais un relatif. On trouve cela assez régulièrement chez Chaucer ; whan pat étant une forme amplifiée de that (sous influence $d u$ français «lorsque»). On remarque aussi la tournure existentielle : ther was/was ther.

\begin{tabular}{|l|l|}
\hline Chaucer, Canterbury Tales, General Prologue & Prologue général, trad. André Crépin \\
\hline With hym ther was a gentil Pardoner & Près de lui trottait un vendeur d'indulgences \\
Of Rouncivale, his freend and his compeer & De l'hôpital Roncevaux, son compère, \\
That streight was comen fro the court of & Tout droit accouru de la Cour de Rome [litt. \\
Rome (669-671) & qui venait tout droit de la cour de Rome] \\
\hline Ne was ther swich another Pardoner. & C'était le meilleur vendeur d'indulgences. \\
For in his male he hadde a pilwe-beer, & Son ballot renfermait une taie d'oreiller \\
Which pat he seyde was oure lady veyl : & Qu'il disait être le voile de Notre-Dame. \\
He seyde he hadde a gobet of the seyl & Il disait avoir un morceau de voile \\
That seint Peter hadde, whan pat he wente & De la barque de Pierre [litt. que Pierre avait], \\
... (693-698) & quand il alla... \\
\hline
\end{tabular}

Which peut s'employer avec un antécédent animé assez longtemps, comme le montrent Our father which art in heaven au XVII ${ }^{\mathrm{e}}$ siècle («Notre père qui êtes aux cieux ») et l'exemple suivant :

Doctor: This disease is beyond my practice: yet I have known those which have walked in their sleep who have died holily in their beds. (Macbeth, V, II)

La distinction who animé et which inanimé n'apparaît pas avant les XVII ${ }^{\mathrm{e}}-\mathrm{XVIII}{ }^{\mathrm{e}}$ siècles. Le relatif $\varnothing$, qui selon Stévanovitch (2008: 76) existe dès le VA et " s'utilise en position sujet jusqu'en anglais moderne ", gagne en fréquence au début de l'AM, mais, considéré comme familier, il décline aux XVIII ${ }^{\mathrm{e}}$ et $\mathrm{XIX}^{\mathrm{e}}$ siècles. La position sujet telle qu'on peut l'observer dans cet exemple n'est plus correcte en $\mathrm{AC}^{14}$ :

Viola: My father had a daughter $\emptyset$ loved a man (Shakespeare, Twelfth Night, II, 4)

\begin{tabular}{|l|l|l|l|l|}
\hline Gender & $\begin{array}{l}\text { Subjective } \\
\text { case }\end{array}$ & $\begin{array}{l}\text { Objective } \\
\text { case }\end{array}$ & $\begin{array}{l}\text { Possessive, } \\
\text { determiner }\end{array}$ & Determiner \\
\hline personal & $\begin{array}{l}\text { which } \rightarrow \text { who } \\
\text { that } \\
\text { (zero) }\end{array}$ & $\begin{array}{l}\text { whom } \\
\text { that } \\
\text { zero }\end{array}$ & whose & which \\
\hline non-personal & $\begin{array}{l}\text { which } \\
\text { that } \\
\text { (zero) }\end{array}$ & $\begin{array}{l}\text { which } \\
\text { that } \\
\text { zero }\end{array}$ & $\begin{array}{l}\text { whose } \\
\text { (whereof) } \\
\text { (of which) })\end{array}$ & which \\
\hline
\end{tabular}

Tableau 9 : Pronoms relatifs en anglais élisabéthain (Nevalainen 2006 : 84)

Selon Fischer \& Wurff (2006 : 128-129), whose/whom/which sont fréquemment employés comme relatifs dès le $\mathrm{XV}^{\mathrm{e}}$ siècle, mais pas who; ils émettent l'hypothèse suivante : « as a nominative it needed no preposition and therefore the relative could be expressed by the usual that, common with both animate and inanimate antecedents all through the ME period and beyond.». Ils ajoutent que comme pronom indéfini/relatif libre il intervenait surtout en position sujet et était peut-être trop fortement généralisant pour fonctionner comme simple relatif (ibid.). Nevalainen (2006: 84) apporte les précisions suivantes :

The relative who is first attested as a subject relative pronoun in the early fifteenth century in closing formulae of letters and prayers with reference to God (. . that knoweth God, who have you in his blessed kepyng, from the Stonor letters; Rydén 1983: 127). Who began to diffuse from divine to human reference towards the end of the 
fifteenth century. Because of its origins, it was first used in relative clauses that provided new information about the referent (non-restrictive relative clauses) but were not required to identify it (as is the case in restrictive relative clauses).

All this I shewed to G. Nonne who semeth very lothe that Dobbes shuld have yt becawse he thynketh he will deale streyghtlye with the tenantes... (CEEC, Francis Wyndham, 1577: BACON, 249)

Nevalainen (ibid.) ajoute qu'au cours de la période élisabéthaine, « the zero strategy lost ground in the subject position and who was established especially in the written language. Who could also appear in the object position instead of whom, although this was less common with the relative who than with the corresponding interrogative pronoun. »

Les relatifs adverbiaux du MA sont ther et wher, qui subsiste aujourd'hui sous la forme where. Whatever (XIV ${ }^{\mathrm{e}} \mathrm{s}$.) n'existait pas sous cette forme, l'équivalent était swā hwcet swā (whatsoever/whatever): "Generalizing relative pronouns can be made from interrogatives in $h w$ - by adding swa ... swa, e.g. swa hwa swa whoever [...]» (Campbell 1991 : 294). Guy Bourquin (2011) pense que dans ces combinaisons (nombreuses avec $s w \bar{a} . . . s w \bar{a})$ le premier élément est déictique et le second anaphorique. Pour rappel, ce terme swā a donné à la fois as et so en AC. On a ici un outil à la fois "relatif » et « indéfini » avec swā hwcet swā.

\section{Les pronoms indéfinis}

Les indéfinis étaient nombreux en VA, presque toujours des formes composées, parmi

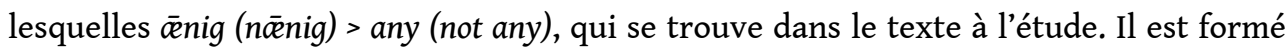
du numéral « un" + suffixe -ig " qui a les caractéristiques de, enclin à, orienté vers, consistant à » (Bourquin $2011: 6$ ).

La valeur du suffixe $-i \dot{g}$ dans $\bar{x} n i \dot{g}(\bar{a} n+-i \dot{g})$ renvoie aux caractéristiques de la classe d'appartenance, appréhendées soit au niveau du parcours indifférenciant ("qui que ce soit, n'importe lequel"), soit lors de l'individuation non encore spécifiée ("un quelconque, un certain, quelque"). Après le vieil-anglais le marqueur any perdra cette dernière valeur au profit de la forme non suffixée ān (> "one") alors que son homologue allemand einig au contraire, ne conservera que celle-ci (au pluriel, einige = "quelquesuns"). L'étymologie du suffixe -ig est difficile à cerner: IE *- $k$ - ? (cf. LAT unicus) ou pourquoi pas * $k w$ - (prédominance du parcours et du renvoi à la classe) ? (Bourquin $2011: 16)$

āwiht, ōwiht, āwuht, ōwiht, āht, ōht = anything; wiht = «être, objet », d'où l'AC wight (= ghost/spirit)

Bourquin (id.) pour ever donne l'étymologie suivante : $\bar{x} f r e<\bar{a}$ in fēore = lit. " toujours dans la vie " (d'une racine IE aiw- vital force, eternity > $\bar{a}$ « temps, éternité »). "The interrogative pronouns in $h w$ - may be used as indefinite pronouns: $h w a$ anyone, someone, hwceper either of two, one or other, hwelc, hwilc anyone " nous dit Campbell (1991: 293), qui donne l'exemple ci-dessous :

sona swa sacerda hwyli hwone ...gesyhp

as soon as of the priests anyone anyone observes 
Eischer \& Wurff (2006: 128) vont dans le même sens, ajoutant que cet usage de l'interrogatif comme indéfini les faisait apparaître comme des relatives libres et qu'elles apparaissaient souvent au style indirect.

61 Campbell (1991) indique également comme pronoms indéfinis courants, eall « all », $\bar{x} l c$ " each ", änig / nōenig, nān " none ", se ilca / se sylfa " the same », entre autres. Par ailleurs il existait un indéfini man " on », forme atone de mann, qui existe toujours en allemand (man sagt : "on dit ») et en néerlandais (men). La forme inaccentuée s'affaiblit en me pendant le $\mathrm{XIV}^{\mathrm{e}}$ siècle et a pratiquement disparu à la fin $\mathrm{du} \mathrm{XV}^{\mathrm{e}}$ siècle. Une des questions qui se pose à ce propos est la suivante: est-ce que man a disparu à cause d'une préférence pour la construction passive, ou bien celle-ci a-t-elle amené la disparition de man - ou les deux? En revanche, l'émergence des passifs prépositionnels avec agent non-spécifié seraient bien dus à la perte de man.

Man brohte his heafod on ánum disce and sealde ðam mēedene

On apporta de lui la tête sur un plat et (on) le donna à la jeune-fille (Judith)

Fischer \& Wurff (2006 : 121) signalent qu'en VA on pouvait avoir sume pa englas (Ælfric's Hom. I, 7 236.147) pour « some of the angels » et notent qu'en MA on tend de plus en plus à insérer of entre un quantifieur et un article défini, ce qui est la norme en AC. "The new of-form is found already in ME after some and any ", écrivent-ils (id.), mais elle ne s'imposera que beaucoup plus tard avec all et both. Les deux auteurs citent Denison (1998 : 117), selon lequel la construction sans of était toujours la plus utilisée au XIXe siècle. Ils ajoutent que le quantifieur pouvait occuper en VA une place variable (ex. some the men ate, the men some ate, the men ate some) et notent :

When the number of slots decreased, probably due to the general fixation of word order in ME, some of these quantifiers or their position disappeared (e.g. the men some was lost but not the men all) while others became reanalyzed (e.g. in some the men, the word some came to be seen as an indefinite pronoun and, consequently of was added). (Fischer \& Wurff $2006: 121$ )

Ainsi des constructions avec prédéterminant sont remplacées par la construction en of.

\section{Conclusion}

Il est évident qu'il y aurait beaucoup encore à dire sur l'évolution des pronoms, notamment au niveau des constructions syntaxiques plus complexes, mais mon propos - à la demande des organisateurs du colloque - était de faire à la fois un état des lieux de la situation des pronoms en vieil-anglais et de l'évolution subséquente. On se rapportera avec profit aux ouvrages cités en bibliographie pour de plus amples recherches. Cependant, on voit que le système des pronoms a considérablement évolué avec la perte des déclinaisons et l'instauration d'un ordre des mots fixe. Le système s'est enrichi avec l'emprunt des pronoms personnels du pluriel au scandinave, et la transformation du génitif des pronoms personnels à la fois en déterminants et pronoms possessifs. Les pronoms interrogatifs ont développé un emploi relatif et le réfléchi en self s'est définitivement installé à la place de l'usage du pronom personnel simple.

65

Il reste un domaine pour lequel mes recherches ont été sommaires, c'est celui de l'apparition des clivées: des structures de ce type semblent être répertoriées en VA, notamment dans des gloses ou des traductions du latin. En MA, on note aussi une influence du français. Mais il semble que le contact avec les substrats celtiques, langues où cette structure est très employée, ait joué un rôle aussi. Selon Filppula (2009), c'est 
très clair pour les clivées : soit influence de type renforcement des structures existantes ou bien influence directe, comme pour certains types de clivées.

\section{BIBLIOGRAPHIE}

Barber, C. (1995)[1993]. The English Language: A Historical Introduction. Cambridge: Cambridge University Press.

Baugh A.C. \& Cable, T. (1989). A History of the English Language. 3rd ed. London: Routledge.

Bourcier, G. (1986). Quelques remarques sur les « indéfinis » en anglais « ancien ». Bulletin des anglicistes médiévistes 29-30.

Bourquin, G. (2011). Les marqueurs du défini et de l'indéfini : tendances évolutives du vieilanglais à aujourd'hui. Bulletin des anglicistes médiévistes 79.

Campbell, A.. (1991)[1959]. Old English Grammar. Oxford: Clarendon Press.

Chevillet, F. (1991). Les variétés de l'anglais. Paris: Armand Colin.

Crépin, A. (1972)[1967]. Histoire de la langue anglaise. Coll. « Que sais-je ?». Paris : Presses Universitaire de France.

Crépin, A. (1978). Grammaire historique de l'anglais. Paris : Presses Universitaire de France.

Crépin, A. (1994). Deux mille ans de langue anglaise. Paris : Nathan Université.

Crystal, D. (1995). The Cambridge Encyclopedia of the English Language. Cambridge : Cambridge University Press.

Crystal, D. (1988)[1990], The English Language. London: Penguin.

Crystal, D. (2004). The Stories of English. London: Penguin.

Denison, D. (1993). English Historical Syntax: Verbal Constructions. London \& New York: Longman.

Denison, D. (1998). Syntax. In Romaine, S. (ed.) The Cambridge History of the English Language. Vol. IV: 1776-1997, 92-329. Cambridge: Cambridge University Press.

Denison, D. (2009). Category change and gradience in the determiner system. In Kemenade \& Los (eds.), 279-304.

Elliott, R. (1984). Thomas Hardy's English. Oxford: Blackwell.

Filppula, M. (2009). The making of Hiberno-English and other "Celtic Englishes". In Kemenade \& Los (eds.), 507-536.

Fischer, O. \& Wurff, W. van der. (2006). Syntax. In Hogg, R. \& Denison, D. (eds.), 109-198.

Hogg, R. \& Denison, D. (eds.) (2006). A History of the English Language. Cambridge: Cambridge University Press.

Kemenade, A. van \& Los, B. (eds.) (2009). The Handbook of The History of English. Malden, MA / Oxford / Chichester: Wiley-Blackwell 
Lancri, A. (1994). Quelques réflexions à propos des relatives en vieil-anglais. Bulletin des anglicistes médiévistes 45.

Lancri, A. (1999). A contrastive analysis of relative clauses in Old English and Middle Egyptian. In Contrast, Comparison and Communication. C.I.E.R.E.C./Travaux XCVI. Saint-Etienne : Publications de l'Université de Saint-Étienne.

Lass, R. (2006). Phonology and morphology. In Hogg \& Denison (eds.), 43-108.

Marsden, R. (2004). The Cambridge Old English Reader. Cambridge: Cambridge University Press.

Mossé, F. (1950-1959). Manuel de l'anglais du Moyen Age. Vol. 1: Vieil-Anglais (1950, 2e éd.), Vol. 2: Moyen-Anglais (1959, 2e éd.). Paris : Aubier-Montaigne.

Nevalainen, T. (2006). An Introduction to Early Modern English. Edinburgh: Edinburgh University Press.

Nevalainen, T. (2009). Historical sociolinguistics and language change. In Kemenade \& Los (eds.), 558-588.

Onions, C. (gen. ed.) (1966). The Oxford Dictionary of English Etymology. Oxford: Clarendon Press.

Pons, E.A. (1956). Cours de philologie anglaise. Tome I : Aspects généraux et vieil-anglais. *Fascicule II : Morphologie historique et indications syntaxiques. $2^{\text {ème }}$ cahier : Morphologie pronominale. Paris : Centre de Documentation Universitaire.

Quirk, R. \& Wrenn, C. L. (1996). An Old English Grammar. 2nd ed. London: Routledge.

Santin-Guettier, A.-M. (2003). Surface et profondeur : le cas de l'opérateur WHAT à la lumière de WHICH et de THAT. In Stévanovitch \& Tixier (dir.), 93-107.

Stévanovitch, C. (2003). Niveaux d'émotion : tutoiement et vouvoiement dans quelques pièces de Shakespeare. In Stévanovitch \& Tixier (dir.), 429-442.

Stévanovitch, C. \& Tixier, R. (dir.) (2003). Surface et profondeur. Mélanges offerts à Guy Bourquin à l'occasion de son $75^{\text {ème }}$ anniversaire. Coll. «Grendel » ${ }^{\circ} 7$. Nancy : Publications de l'AMAES.

Stévanovitch, C. (2006). Le sujet de troisième personne dans la poésie vieil-anglaise. Bulletin des anglicistes médiévistes 69.

Stévanovitch, C. (2008). Manuel d'histoire de la langue anglaise. Des origines à nos jours. Paris : Ellipses. Watkins, C. (ed.) (1985). The American Heritage Dictionary of Indo-European Roots. Boston: Houghton Mifflin Company.

Wright, J. (1961). Old English Grammar. 3rd ed. Oxford: Oxford University Press.

\section{ANNEXES}

\section{Annexe: Quatre versions différentes d'un même épisode biblique « Le fils prodigue » (Luc XV, 11-15)}

\section{VA (IXe siècle)}

Sōðlīce sum mann hæfde twēgen suna ; ðā cwæð sē gingra tō his fæder : 'Fæder, sele mēe mīnne dæl mīnre æhte, ðe mē tō ge byreð.' Đā dælde hē him his æhta. Đá æfter feawum 
dagum eall his ðing ge gaderode sē gingra sunu, and férde wræclīce on fierlen rīce, and for spilde ðær his æhta libbende on his gælsan. Đā hē hie hæfde ealle ā mierrede, ðā wearð micel hungor on ðæm rīce, and he wearð wædla. Đā fērde hē and folgode ānum burg-sittendum menn ðæs rīces; ðā sende hē hine to his tūne, ðæt hē heolde his swīn.

\section{Wyclif (2 $2^{\text {ème }}$ moitié du XIVe siècle)}

11 Forsothe he seith, Sum man hadde tweye sones;

12 And the 3ongere seide to the fadir, Fadir, 3yue to me the porcioun of substaunce, that byfallith to me. And the fadir departide to him the substaunce.

13 And not aftir manye dayes, alle thingis gederid to gidre, the zongere sone wente in pilgrymage in to a fer cuntree; and there he wastide his substaunce in lyuynge leccherously.

14 And aftir that he hadde endid alle thingis, a strong hungir was maad in that cuntree, and he bigan to haue nede.

15 And he wente, and cleuyde to oon of the citeseyns of that cuntree. And he sente him in to his toun, that he schulde feede hoggis.

\section{Tindale ( $1^{\text {ère }}$ moitié du $X \mathrm{Vl}{ }^{\mathrm{e}}$ siècle)}

11 And he sayde, A certayne man had two sonnes;

12 And the yonger of them sayde to his father, Father, geve me my parte off the goodes, that to me belongeth. And he devided vnto them his substaunce.

13 And not longe after, the yonger sonne gaddered all that he had to gedder, and toke his iorney into a farre countre; and there he wasted his goodes with royetous livinge.

14 And when he had spent all that he had, there rose a greate derth thorow out all that same londe, and he began to lacke.

15 And he went, and clave to a citesyn of that same countre. Which sent hym to the felde, to kepe his swyne.

\section{Authorized version (1611)}

11 And he said, A certain man had two sons :

12 And the younger of them said to his father, Father, give me the portion of goods that falleth to me. And he divided unto them his living.

13 And not many days after the younger sonn gathered all together, and took his journey into a far country, and there wasted his substance with riotous living.

14 And when he had spent all, there arose a mighty famine in that land; and he began to be in want.

15 And he went and joined himself to a citizen of that country ; and he sent him into his fields to feed swine. 


\section{NOTES}

1. Les sigles suivants seront utilisés dans l'article $: \mathrm{VA}=$ vieil-anglais, $\mathrm{MA}=$ moyen-anglais, $\mathrm{AE}=$ anglais élisabéthain, $\mathrm{AC}=$ anglais contemporain, $\mathrm{VN}$ = vieux norrois (scandinave).

2. Edition utilisée : The Bible with Apocrypha: Authorized King James Version. Oxford Crown Edition. Oxford University Press.

3. Grande Mutation Vocalique : fermeture générale des voyelles au MA jusque vers 1500 .

4. Je remercie Jean-Marc Gachelin de m'avoir indiqué cette référence.

5. Pour plus de précisions, voir Stévanovitch 2003 (notamment son analyse des PP dans King Lear).

6. Voir également «Choosing thou or you », Crystal (2004), p. 307-310.

7. Voir également les poèmes de William Barnes.

8. Jean-Marc Gachelin me signale que cette forme (') em existe dans de nombreux dialectes, comme survivance du VA him, MA h(e)om (cas régime) et non comme corruption de them (lettre personnelle). Voir aussi Chevillet (1991) sur ce point.

9. Pons (1956: 277) les appelle démonstratifs « rapprochés».

10. Il existait de nombreuses formes dialectales en MA, pour le détail des formes de l'article on pourra se reporter à Mossé (1959: 91).

11. Lych-gate : « porche de cimetière ", traditionnelle entrée d'un cimetière anglais.

12. On la retrouve dans des locutions : for porm pe " parce que », mid prem pe « pendant que ».

13. Complémenteur ici.

14. Exception faite de structures du type There's a bloke Ø wants to see you, dans la langue parlée ou dialectale.

\section{RÉSUMÉS}

Le but de cet article est de faire tout d'abord un état des lieux des pronoms existant en vieilanglais, puis d'étudier leur évolution lors des périodes moyen-anglaise et élisabéthaine. Sont étudiés les pronoms personnels, les pronoms démonstratifs, les pronoms interrogatifs, ainsi que l'évolution de ces derniers vers leur utilisation comme pronoms relatifs et l'émergence des pronoms possessifs à partir du génitif du pronom personnel. Cet article traite un ensemble de données au niveau morphologique, phonétique, sémantique et syntaxique.

The aim of this article is to give an account of the evolution of pronouns from the Old English period to the Renaissance. It starts with a description of the Old English system (personal, demonstrative, interrogative and relative pronouns) and examines the changes occurring in the Middle English and Elizabethan periods with the emergence of possessive pronouns stemming from the Genitive case of the personal pronouns and the increasing use of the interrogative pronouns as relatives. The article thus studies the pronouns from phonetic, morphological, semantic and syntactic points of view. 
INDEX

Mots-clés : pronom, vieil-anglais, moyen-anglais, période élisabéthaine

Keywords : pronoun, Old English, Middle English, Elizabethan period

\section{AUTEUR}

\section{CATHERINE DELESSE}

Université de Lorraine 